Proceedings of the II Symposium on Applied Nuclear Physics and Innovative Technologies, Kraków, September 24-27, 2014

\title{
INDRA: A $4 \pi$ Detector for Nuclear Dynamics and Thermodynamics Studies
}

\author{
M.F. RIVET*for the INDRA Collaboration \\ Institut de Physique Nucléaire, CNRS/IN2P3, Université Paris-Sud 11, 91406 Orsay, France
}

\begin{abstract}
This paper reports on some topics presently studied by the INDRA collaboration in nuclear dynamics and thermodynamics. We shall show information on the density dependence of the symmetry term of the nuclear equation of state. A possible origin of multifragmentation and fragment formation will be demonstrated. Finally we report on an experiment aiming at determining the influence of isospin on the nucleus level density.
\end{abstract}

DOI: $10.12693 /$ APhysPolA.127.1552

PACS: 25.70.Mn, 25.70.Pq, 24.60.Ky

\section{Introduction}

Nuclear thermodynamics study the properties of an equilibrated nuclear system, that may be excited (temperature $T>0$ ), compressed or diluted, with a high angular momentum. The nuclear interaction is formally similar to the van der Waals force acting on classical fluids. Nuclear systems are thus expected to undergo phase transitions. However nuclei are finite, which leads to specific behaviours in the transition region. Multifragmentation is seen as the manifestation of the liquid-gas type phase transition [1].

Nuclear dynamics deals with transport properties of nuclear matter, such as dissipation, mass and isospin transfer. These properties depend on the incident energy, and on the impact parameter of the collisions $[2,3]$. The ultimate goal of dynamics and thermodynamics studies is to establish the equation of state (EOS) of nuclear matter, that is poorly known for $N / Z \neq 1$.

Heavy-ion nuclear collisions allow to drive nuclear systems towards extreme states in density, temperature, isospin $(N / Z)$ or angular momentum. At incident energies ranging from 10 to $100 \mathrm{AMeV}$ charged product multiplicities are high, which requires a detection system covering the whole (phase)space. Such is the chargedproduct array INDRA: it covers $90 \%$ of $4 \pi$, comprises 336 detection modules, and has low detection thresholds [4]. It is particularly efficient for studying central collisions, and the projectile remnants of semi-peripheral collisions. In the following we report on three topics illustrating the studies made by the INDRA collaboration.

\section{Search for symmetry term of the nuclear EOS}

The energy per nucleon, $\varepsilon=\frac{E}{A}$, (i.e. the EOS) in nuclear matter can be written as

$$
\varepsilon(\rho, I)=\varepsilon(\rho, I=0)+\varepsilon_{\mathrm{sym}}(\rho) I^{2}
$$

with

$$
I=\frac{\rho_{\mathrm{n}}-\rho_{\mathrm{p}}}{\rho}=\frac{N-Z}{A} .
$$

\footnotetext{
* corresponding author; e-mail: rivet@ipno.in2p3.fr
}

While it is relatively well established for symmetric matter at normal density, its evolution with density is poorly constrained for asymmetric nuclear matter. Many groups worldwide are presently working on the subject; particularly several experimental data are published that try to constrain the shape of the symmetry term. One of the experimental method is the study of isospin transport in semi-peripheral heavy-ion collisions. The INDRA collaboration obtained data for two systems ${ }^{124} \mathrm{Xe}+{ }^{124} \mathrm{Sn}$ and ${ }^{136} \mathrm{Xe}+{ }^{112} \mathrm{Sn}$, at $32 \mathrm{AMeV}$. Note that the total system is identical in both cases. The fragment multiplicity, that depends on the isospin, is followed as a function of the impact parameter.

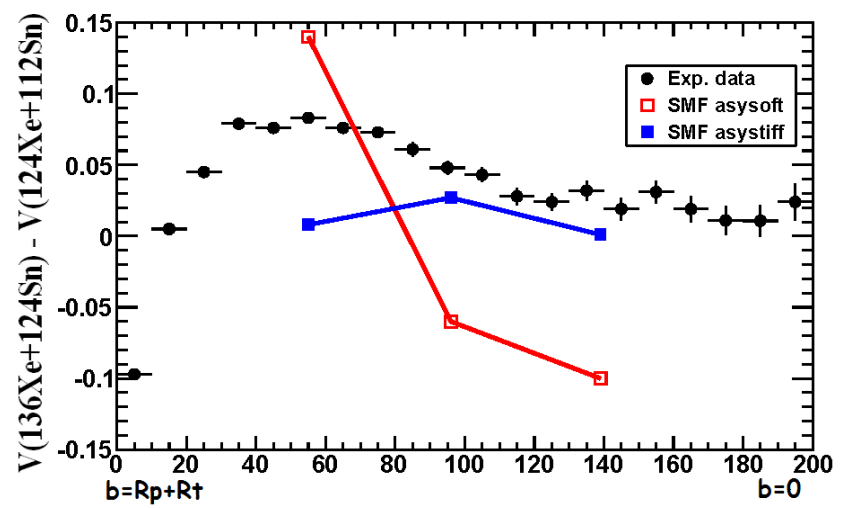

Fig. 1. Evolution of the difference of forward emitted fragment $(Z \geq 3)$ multiplicities for the systems ${ }^{124} \mathrm{Xe}+{ }^{124} \mathrm{Sn}$ and ${ }^{136} \mathrm{Xe}+{ }^{112} \mathrm{Sn}$, as a function of the impact parameter. The abscissa scale refers to the transverse energy of light charged particles, used as an impact parameter selector from $b \sim R_{p}+R_{t}$ (left) to $b \sim 0$ (right). Lines show results of the SMF model. Adapted from [5].

Figure 1 presents the obtained evolution of the difference between the fragment multiplicities of the two systems. Data are compared with results from a stochastic mean field simulation (SMF) [6], with two different implementations of the potential symmetry energy: an asystiff, that linearly evolves with density, and an asysoft that has a maximum close to normal density. The first 
one well matches data for impact parameters below $6 \mathrm{fm}$. Thus in the SMF framework, we state that, at subsaturation density, the density dependence of the symmetry energy is linear [5].

\section{Multifragmentation and fragment formation}

A long debated question is the origin of multifragmentation and the mechanism of fragment formation. In molecular dynamics models fragments survive initial state correlations and can be early identified, when the system is close to normal density. Conversely in SMF model the system is first compressed, then evolves to a low density state in the spinodal region, in which density fluctuations end up in fragments (liquid) and particles (gas phase). The universal fluctuation model, developed some years ago, can provide pertinent information on the relationship between fragment formation and phase transition by looking at the properties of the fragments. Analysing central $\mathrm{Xe}+\mathrm{Sn}$ collisions between 25 and $100 \mathrm{AMeV}$, the INDRA collaboration found a $\Delta$ scaling law for the charge of the largest fragment of multifragmentation partitions, $Z_{\max }$ [8]. Simply written this law implies that the variance and the mean value of the $Z_{\max }$ distributions follow : $\sigma^{2} \sim\langle m\rangle^{2 \Delta}$.

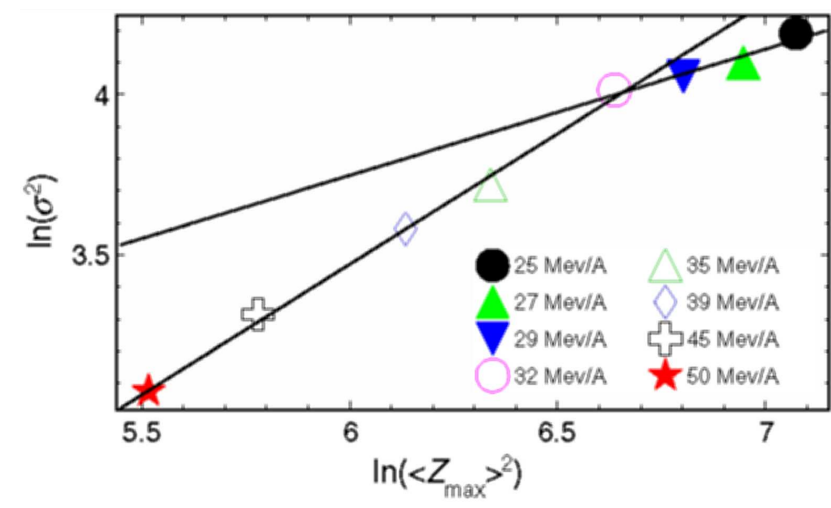

Fig. 2. Log-log correlation between the first two cumulant moments $\left(\langle Z \max \rangle^{2}\right.$ and $\left.\sigma^{2}\right)$ of the order parameter distribution; Lines represent linear fits performed in the range 50-32 $A \mathrm{MeV}$ (respectively 32-25 $A \mathrm{MeV}$ ) which correspond to a slope $\Delta \sim 1$ (respectively $\Delta \sim 1 / 2$ ) From [7].

Figure 2 shows that the value of $\Delta$ changes from $1 / 2$ to 1 around $32 \mathrm{AMeV}$. This implies that the system evolves from an ordered to a disordered phase with increasing energy, while the scaling of $Z_{\max }$, and not of the fragment multiplicity, signs the fragment formation as an aggregation scenario. This result is compatible with multifragmentation originating from density (and isospin) fluctuations in the spinodal region [1].

\section{4. $N / Z$ dependence of the level density parameter}

Excited fragments formed in the multifragmentation process may have an exotic isospin. The final fragment distributions are compared with results of statistical de-excitation models, in which a fundamental ingredient is the level density parameter, $a$ - related to the EOS. The isospin dependence of $a$ far from the stability line is not known, and different extrapolations lead to very different predictions [10]. In the hope to get new information the INDRA collaboration performed at GANIL/SPIRAL a fully exclusive experiment, in which the evaporation residues (ER) were completely identified in the VAMOS spectrometer [11], whereas all coincident light charged particles were detected in INDRA. Five compound nuclei excited at $\approx 2.9 \mathrm{AMeV}$ were formed, 92,94,96,100,104 Pd.
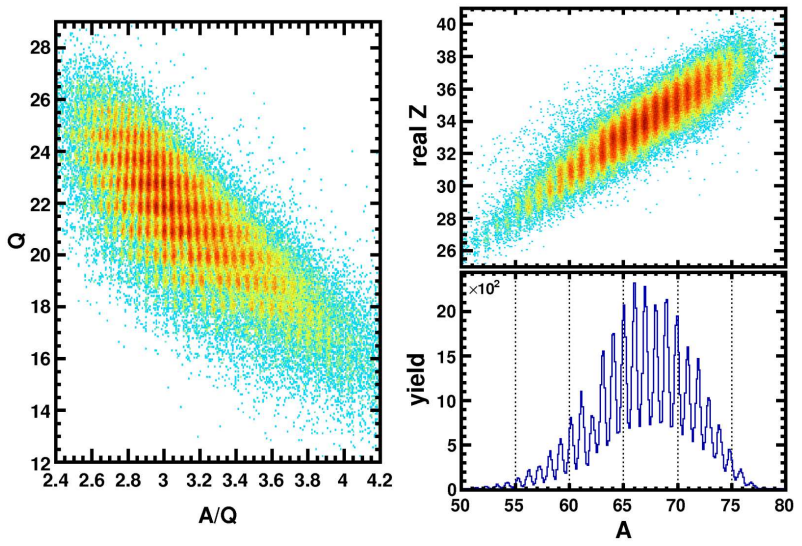

Fig. 3. Charge state $Q$ vs. $A / Q, A-Z$ map and $A$ distribution measured in the VAMOS spectrometer at $0^{\circ}$ for a given $B \rho$. The system is ${ }^{36} \mathrm{Ar}+{ }^{58} \mathrm{Ni}$. From [9].

Besides obtaining ER charge and mass distributions (Fig. 3), particle multiplicities and energy spectra, we will be able to weight the different chains leading to a given ER. Neutron multiplicity can be derived when the total system charge has been detected. This unique set of information should test the isospin dependence of $a$ and strongly constrain the statistical de-excitation codes.

\section{Perspectives}

In the mid-term our goal is to experimentally deepen our studies of isospin effects on nuclear dynamics and thermodynamics. In that aim some forward rings of INDRA will be substituted by the FAZIA demonstrator [12], to improve granularity and isotopic identification. Exotic (and particularly neutron-rich) beams, accelerated to some tens of $\mathrm{MeV} /$ nucleon, are strongly required.

\section{References}

[1] B. Borderie, M.F. Rivet, Prog. Part. Nucl. Phys. 61, 551 (2008).

[2] V. Baran, M. Colonna, V. Greco, M. Di Toro, Phys. Rep. 410, 335 (2005).

[3] Bao-An Li, Lie-Wen Chen, Che Ming Ko, Phys. Rep. 464, 113 (2008). 
[4] J. Pouthas, B. Borderie, B. Borderie , R. Dayras, E. Plagnol, M.F. Rivet, F. Saint-Laurent, J.C. Steckmeyer, G. Auger, C.O. Bacri, S. Barbey, A. Barbier, A. Benkirane, J. Benlliure, B. Berthier, E. Bougamont, P. Bourgault, P. Box, R. Bzyl, B. Cahan, Y. Cassagnou, D. Charlet, J.L. Charvet, A. Chbihi, T. Clerc, N. Copinet, D. Cussol, M. Engrand, J.M. Gautier, Y. Huguet, O. Jouniaux, J.L. Laville, P. Le Botlan, A. Leconte, R. Legrain, P. Lelong, M. Le Guay, L. Martina, C. Mazur, P. Mosrin, L. Olivier, J.P. Passerieux, Nucl. Instrum. Methods Phys. Res. A 357, 418 (1995).

[5] G. Ademard, B. Borderie, A. Chbihi, O. Lopez, P. Napolitani, M.F. Rivet, M. Boisjoli, E. Bonnet, R. Bougault, J.D. Frankland, E. Galichet, D. Gruyer, D. Guinet, M. Kabtoul, G. Lehaut, P. Lautesse, M. La Commara, N. Le Neindre, P. Marini, M. Pârlog, P. Pawłowski, E. Rosato, R. Roy, G. Spadaccini, E. Vient, M. Vigilante, J.P. Wieleczko, Eur. Phys. J. A 50, 33 (2014).

[6] M. Colonna, M. Di Toro, A. Guarnera, S. Maccarone, M. Zielinska-Pfabé, H.H. Wolter, Nucl. Phys. A 642, 449 (1998).

[7] D. Gruyer, J. Frankland, R. Botet, M. Płoszajczak, E. Bonnet, A. Chbihi, G. Ademard, M. Boisjoli, B. Borderie, R. Bougault, D. Guinet, P. Lautesse, L. Manduci, N. Le Neindre, P. Marini, P. Pawłowski, M.F. Rivet, E. Rosato, G. Spadaccini, M. Vigilante, J.P. Wieleczko, Phys. Rev. Lett. 110, 172701 (2013).
[8] J.D. Frankland, A. Chbihi, A. Mignon, M.L. Begemann-Blaich, R. Bittiger, B. Borderie, R. Bougault, J.-L. Charvet, D. Cussol, R. Dayras, D. Durand, C. Escano-Rodriguez, E. Galichet, D. Guinet, P. Lautesse, A. Le Févre, R. Legrain, N. Le Neindre, O. Lopez, J. Łukasik, U. Lynen, L. Manduci, J. Marie, W.F.J. Müller, L. Nalpas, H. Orth, M. Pârlog, M. Pichon, M.F. Rivet, E. Rosato, R. Roy, A. Saija, C. Schwarz, C. Sfienti, B. Tamain, W. Trautmann, A. Trzcinski, K. Turzó, A. Van Lauwe, E. Vient, M. Vigilante, C. Volant, J. P. Wieleczko, B. Zwieglinski, Phys. Rev. C 71, 034607 (2005).

[9] G. Ademard, L. Augey, et al. (INDRA Collaboration), in: Proc. Int. Workshop on Multifacets of EoS and Clustering 2014, Eds. B. Borderie, R. Bougault, et al., Catania (Italy), 2014, EPJ Web of Conferences.

[10] S.I. Al-Quraishi, S.M. Grimes, T.N. Massey, D.A. Resler, Phys. Rev. C 67, 015803 (2003).

[11] S. Pullanhiotan, M. Rejmund, A. Navin, W. Mittig, S. Bhattacharyya, Nucl. Instrum. Methods Phys. Res. A 593, 343 (2008).

[12] R. Bougault, G. Poggi, et al. (FAZIA Collaboration), Eur. Phys. J. 50, 47 (2014). 